\title{
A review of the use of propensity score diagnostics in papers published in high- ranking medical journals
}

\author{
Emily Granger ${ }^{1 *}$, Tim Watkins ${ }^{2}$, Jamie C. Sergeant ${ }^{1,3}$ and Mark Lunt ${ }^{1}$
}

\begin{abstract}
Background: Propensity scores are widely used to deal with confounding bias in medical research. An incorrectly specified propensity score model may lead to residual confounding bias; therefore it is essential to use diagnostics to assess propensity scores in a propensity score analysis. The current use of propensity score diagnostics in the medical literature is unknown. The objectives of this study are to (1) assess the use of propensity score diagnostics in medical studies published in high-ranking journals, and (2) assess whether the use of propensity score diagnostics differs between studies (a) in different research areas and (b) using different propensity score methods.

Methods: A PubMed search identified studies published in high-impact journals between Jan 1st 2014 and Dec 31 st 2016 using propensity scores to answer an applied medical question. From each study we extracted information regarding how propensity scores were assessed and which propensity score method was used. Research area was defined using the journal categories from the Journal Citations Report.

Results: A total of 894 papers were included in the review. Of these, 187 (20.9\%) failed to report whether the propensity score had been assessed. Commonly reported diagnostics were $p$-values from hypothesis tests (36.6\%) and the standardised mean difference (34.6\%). Statistical tests provided marginally stronger evidence for a difference in diagnostic use between studies in different research areas $(p=0.033)$ than studies using different propensity score methods $(p=0.061)$.

Conclusions: The use of diagnostics in the propensity score medical literature is far from optimal, with different diagnostics preferred in different areas of medicine. The propensity score literature may improve with focused efforts to change practice in areas where suboptimal practice is most common.
\end{abstract}

Keywords: Covariate balance, Confounding, Propensity scores, Diagnostics, Epidemiology

\section{Background}

Whilst randomised controlled trials (RCTs) are the gold standard for evaluating treatment effects, they are often infeasible due to time, cost or ethical constraints. In such situations, observational data may provide valuable

\footnotetext{
* Correspondence: emily.granger-2@postgrad.manchester.ac.uk

${ }^{1}$ Centre for Epidemiology Versus Arthritis, Centre for Musculoskeletal Research, Division of Musculoskeletal and Dermatological Sciences, School of Biological Sciences, Faculty of Biology, Medicine and Health, University of Manchester, Manchester M13 9PT, UK

Full list of author information is available at the end of the article
}

information. Unfortunately, observational data analyses are subject to confounding bias. This occurs when patient characteristics that influence the outcome have unbalanced distributions across treatment groups. Any differences observed in the outcome between treatment groups may be partly due to the differences in patient characteristics.

Traditionally, multivariable regression is used to account for the differences in patient characteristics between treatment groups. However, this approach is not

C C The Author(s). 2020 Open Access This article is licensed under a Creative Commons Attribution 4.0 International License, which permits use, sharing, adaptation, distribution and reproduction in any medium or format, as long as you give appropriate credit to the original author(s) and the source, provide a link to the Creative Commons licence, and indicate if changes were made. The images or other third party material in this article are included in the article's Creative Commons licence, unless indicated otherwise in a credit line to the material. If material is not included in the article's Creative Commons licence and your intended use is not permitted by statutory regulation or exceeds the permitted use, you will need to obtain permission directly from the copyright holder. To view a copy of this licence, visit http://creativecommons.org/licenses/by/4.0/ The Creative Commons Public Domain Dedication waiver (http://creativecommons.org/publicdomain/zero/1.0/) applies to the data made available in this article, unless otherwise stated in a credit line to the data. 
always suitable. For example, when the study outcome is binary, a rule of thumb suggests that 10 events should be observed per covariate included in the regression model [1]. This could be infeasible if the outcome is rare and there are many covariates to adjust for. Propensity scores provide a potential solution to this problem. Rosenbaum and Rubin [2] first introduced the propensity score, defined as the probability of treatment assignment conditional on baseline characteristics. Additionally, they demonstrated that conditioning on the propensity score will balance the distribution of characteristics between treatment groups, reducing the chance of confounding bias. Propensity scores are useful for situations with rare binary outcomes because adjusting for the propensity score only is sufficient to improve balance on the measured covariates. They are also useful in situations where the relationship between covariates and treatment is better understood than the relationship between covariates and outcome, since treatment is modelled rather than outcome. Additionally, comparing propensity score distributions between treatment groups can help identify areas of non-overlap in covariate distributions, which are often overlooked when using traditional regression methods [3]. However, it is important to note that propensity scores cannot account for unmeasured confounding: balance will only be improved on covariates used to estimate the propensity score.

Most commonly, propensity scores are estimated using logistic regression. Treatment assignment is regressed on baseline characteristics and the predicted probabilities are the estimated propensity scores. Assuming no unmeasured confounding and no misspecification of the propensity score model, unbiased estimates of treatment effects can be obtained using one of four techniques: matching, stratification, weighting or covariate adjustment. We briefly describe these techniques here, but readers are referred elsewhere for more details [2, 4-9]. Matching involves forming matched sets of treated and control patients, on the basis of having similar propensity scores. Stratification involves dividing patients into equally sized strata based on their propensity score and weighting involves assigning propensity-based weights to each patient. Estimated treatment effects can then be obtained by comparing outcomes in the matched set, within strata (an overall estimate can be obtained by pooling the strata-specific estimates) or in the weighted sample. Finally, covariate adjustment is implemented by including the propensity score as a covariate when regressing outcome on treatment. Each of these techniques aim to balance patient characteristics between treatment groups, but misspecification of the propensity score model could prevent achieving adequate balance, thereby leading to residual confounding bias. Hence, an essential step of propensity score implementation is using appropriate diagnostics to assess the propensity score and ensure that it has adequately reduced confounding bias. Many authors [10-17] have made recommendations regarding appropriate use of diagnostics. More specifically, they recommended against the use of hypothesis tests comparing covariate means or proportions and advocated using standardised differences.

Despite their introduction in 1983, propensity scores were not commonly applied in the medical literature until around 20 years later. More recently, they have become increasingly popular [10]. In the last decade (2007-2017) the number of articles returned from searching 'propensity scores' in PubMed more than tripled over each 5 year period. Following the increase in use of propensity scores, a number of reviews $[10,11$, 18-25] assessing their implementation were published. Regrettably, each review found that propensity score implementation was suboptimal, particularly regarding the use of diagnostics. Many authors were not reporting the use of any propensity score diagnostic, and those who did were often using hypothesis tests, which are widely discouraged. If appropriate diagnostics are not used to demonstrate the balance of potential confounders achieved by the propensity score, readers of the research have no basis for trusting the results. Of the existing reviews on the propensity score literature, only three [11, $19,21]$ consider articles from all areas of medicine, and these collectively include articles published up to 2012 . Since 2012, there has been numerous publications providing guidance on the use of propensity score diagnostics [10-12, 14-17], or proposing new propensity score diagnostics [26-29]. Considering these recent developments in methodology and guidance on practice, the use of propensity score diagnostics in recent medical studies may have improved. Therefore the aim of this review is to update the literature on diagnostic use, but with a focus on high-ranking journals. Such journals could be considered more influential as they are often looked towards as a beacon of best practice. Furthermore, it may beneficial to know which types of studies are more or less likely to report use of suboptimal diagnostics. This information could help us to identify pockets of good practice and areas where efforts to change practice should be focused. Bearing this in mind, the objectives of this review are to: (1) assess the use of propensity score diagnostics in medical studies published in highranking journals and (2) compare use of diagnostics between studies (a) in different research areas and (b) using different propensity score methods.

\section{Methods}

Search strategy

A PubMed search was conducted on 13th November 2017 to identify articles using propensity scores. We 
searched for articles with "propensity score" or "propensity matched" in the title, abstract or as a Medical Subject Heading (MeSH). The search was limited to publications between 2014 and 2016 and to journals which [1] were ranked in the top 10 by the 2013 Journal Citation Report (JCR) impact factor in any JCR medicine category and [2] had a JCR impact factor of at least 4 (the full text search string is given in Additional file 1).

\section{Study selection}

Studies which used propensity scores to answer an applied medical question were included. This includes studies which aim to assess the effect of a health intervention (e.g. drugs or surgical intervention), or the effect of an exposure (e.g. alcohol), on a health related outcome. Studies were excluded if they were methodological, editorials, reviews or letters. Titles and abstracts were screened to assess eligibility.

\section{Data extraction}

Eligible studies were checked manually by one author (EG) to extract information regarding which diagnostic was used to assess the propensity score and which method was used to condition on the propensity score. For eligible journals, the research area and impact factor was obtained from the JCR website (https://webofknowledge.com).

To assess the reliability of the manual search for data extraction, an automatic full-text search was conducted by a second author (TW) using FileLocator Pro to identify which of the included articles used the 'standardised difference', 'c-statistic' or the 'Hosmer-Lemeshow test' as a propensity score diagnostic. These diagnostics were selected because the variety of terms which could be used to refer to these diagnostics is limited compared to other diagnostics (e.g. a t-test may be referred to as hypothesis test, significance test, testing equality of means etc.). For any discrepancies between the two data extraction methods, the article in question was manually checked (EG). Most discrepancies (135/147; 92\%) were due to the full-text search either incorrectly selecting articles which used the diagnostic for something other than propensity scores, or incorrectly omitting articles where the authors had referred to the diagnostic using different terminology.

\section{Data analysis}

Multinomial logistic regression was used to investigate whether or not there were differences in diagnostic use between (a) studies in different research areas and (b) studies using different propensity score methods. In both models, the outcome was a categorical variable indicating which diagnostic was used: (1) hypothesis tests (2) standardised differences (3) c-statistic, (4) the HosmerLemeshow test (5) eye-balling the data (i.e. informally assessing balance by scanning the values in a table or figure comparing covariate means between treatment groups), (6) other and (7) failed to report diagnostic use. The 'other' category comprised of diagnostics that were rarely observed ( $<4 \%$ studies). The independent variables were indicators for either research area or propensity score method and the categories used are the same as those presented in Figs. 2, 3 and 4. Studies which used multiple diagnostics were included in the model once for each diagnostic used and this was accounted for by using a robust standard error estimator which accounts for non-independent observations [30].

The $p$-values associated with each model's F-statistic were reported as an informal measure of the strength of evidence for differences in diagnostic use between

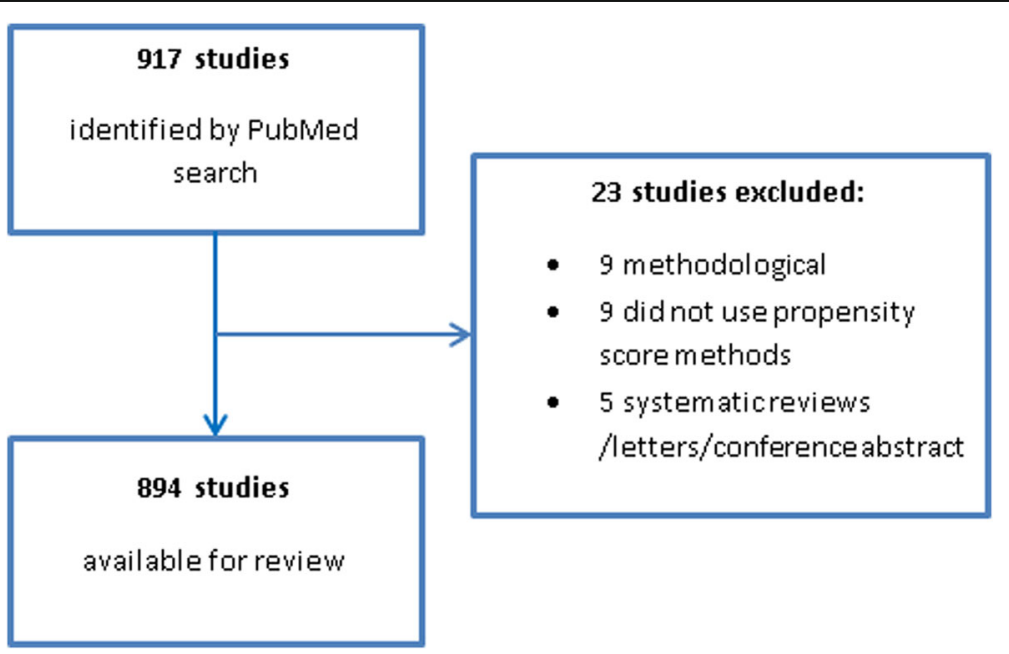

Fig. 1 Flowchart of study selection 


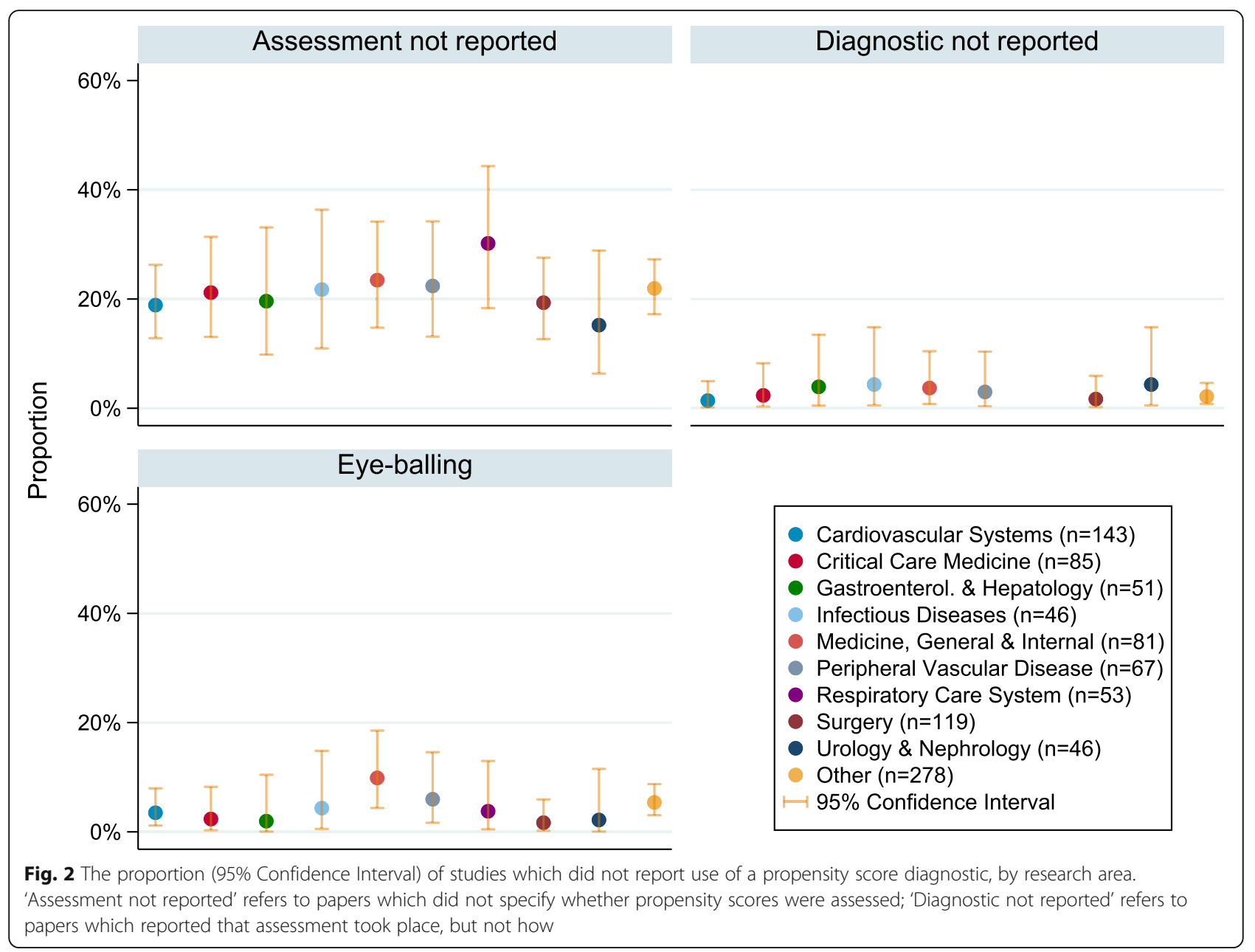

research areas and studies using different propensity score methods. To further investigate possible differences in diagnostic use, we reported the proportion (and associated $95 \%$ confidence interval) of studies using each diagnostic by research area and propensity score method. The 95\% confidence intervals for proportions were calculated using the following formula: $f^{-1}\left\{\ln \frac{\hat{p}}{1-\hat{p}}\right.$ $\left.\pm t_{1-} \alpha / 2,9 \frac{\hat{s}}{\hat{p}(1-\hat{p})}\right\}$

where $f^{-1}(y)=\frac{e^{y}}{1+e^{y}}, \hat{p}$ and $\hat{s}$ are estimates of the proportion and associated standard error respsecitvely, and $t_{1-\alpha / 2, \vartheta^{,}}$is the $(1+\alpha / 2)^{\text {th }}$ quantile of Student's $t$ distribution with $\vartheta$ degrees of freedom. The logit transformation of the confidence interval was used to ensure that the limits lay between 0 and 1.

\section{Results}

The PubMed search identified 917 studies, of which 23 did not meet the inclusion criteria (Fig. 1). The remaining 894 studies were included in the review (a list of the included studies is given in Additional file 2).

Of the 894 studies included in the review, 187 (20.9\%) failed to report whether propensity score diagnostics were used. A further 21 (2.3\%) reported that the propensity score had been assessed, but did not report how. Where diagnostics were reported, the most common were: $p$-values from hypothesis tests (36.6\%), standardised difference (34.6\%), c-statistic (15.4\%) and the Hosmer-Lemeshow test (5.7\%). Eye-balling the data after adjustment to assess balance was reported in (4.3\%) studies. These percentages total to more than 100 since many authors used multiple diagnostics.

The average impact factor (median [interquartile range]) was highest among articles where balance was assessed by eye-balling the data $(9.3[6.0,14.9])$. Ordering the remaining diagnostic categories by median impact factor gives: propensity score assessed, but diagnostic not reported $(7.2[6.0,12.5])$, standardised difference $(7.0[5.6,12.5])$, c-statistic $(7.0[5.5,11.9])$, Hosmer-Lemeshow test $(7.0[5.4,10.4]) p$-values $(7.0$ $[5.2,9.8])$, did not report diagnostic use $(7.0[5.3,12.0])$ 

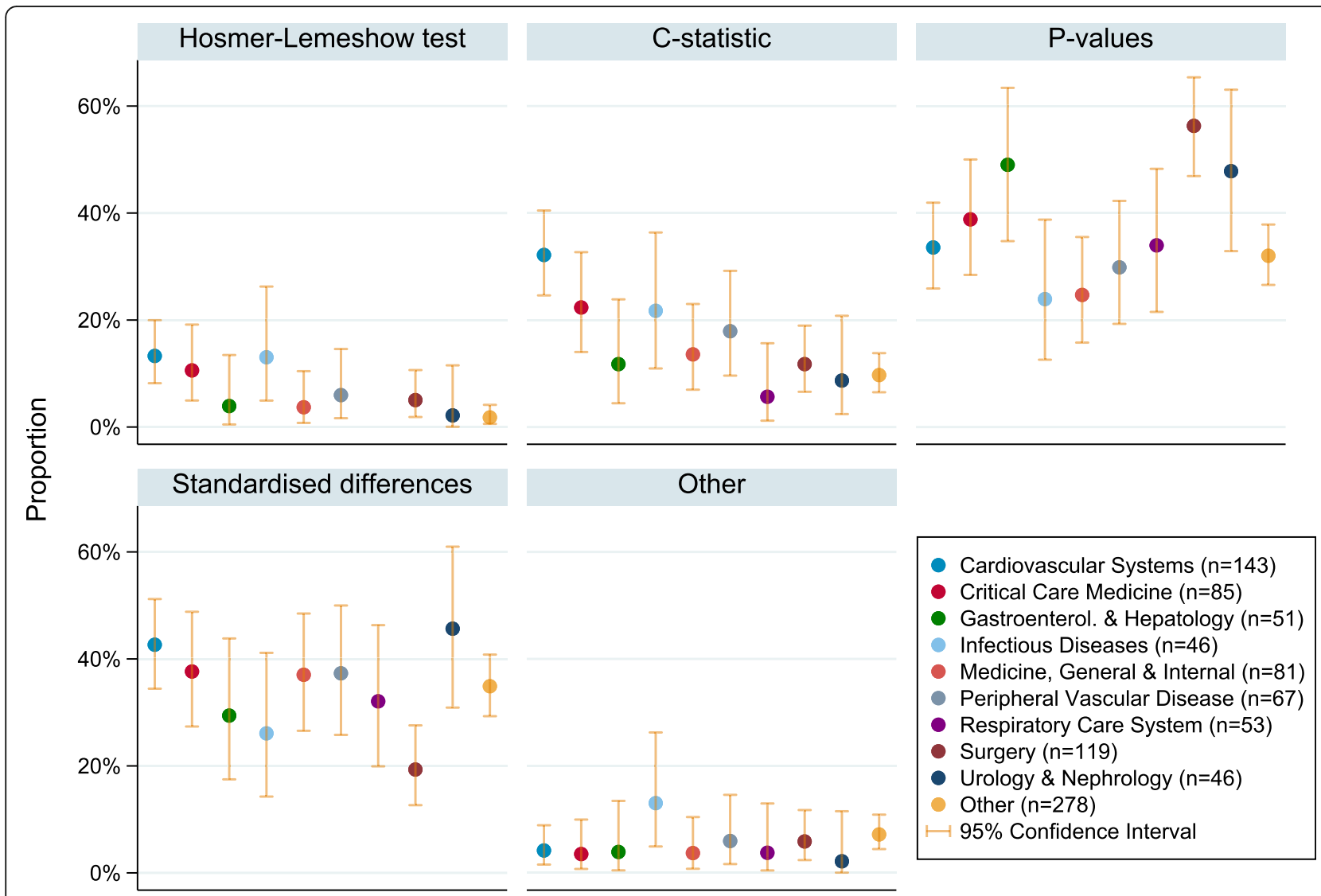

Fig. 3 The proportion (95\% Confidence Interval) of studies using each diagnostic, by research area. 'Other' includes: absolute differences, graphical approaches, post-matching c-statistic, regression, standardised bias, and variance ratios

and other $(6.1[5.3,10.7])$. The similarity in these median indicates no discernible association between diagnostic and impact factor.

\section{Comparison of diagnostics between different research areas}

The most common research areas (defined as areas containing $5 \%$ or more of the total number of studies) are listed in Figs. 2 and 3. There were 25 additional research areas; these have been grouped together into an 'other' category. The number of studies in each of the additional research areas was between $1(0.11 \%)$ and $36(4.03 \%)$. More details on the additional research areas and how many studies were in area is given in Additional file 3 . For the most common research areas, the proportion of studies which either: (1) did not report whether balance assessment took place, (2) reported that balance assessment took place, but did not report which diagnostic was used or (3) relied on eyeballing the data for balance assessment, are given in Fig. 2, along with 95\% confidence intervals. The equivalent proportions and $95 \%$ confidence intervals for studies using: the
Hosmer-Lemeshow test, c-statistic, hypothesis tests, standardised differences or "other" are given in Fig. 3. The F-statistic associated with the multinomial regression model of diagnostic on research area had $p$ value $=0.033$, suggesting a possible difference in diagnostic use between research areas.

Across all research areas, studies in respiratory care had the highest proportion of papers failing to report whether or not diagnostics were used (30\% [95\% CI, 18$44 \%]$ ). In the remaining research areas, between 15 and $23 \%$ of studies failed to report this information.

There is little evidence for a difference in the use of eye-balling or Hosmer-Lemeshow test between research areas (as indicated by the overlapping confidence intervals), however there were some differences in the use of c-statistics, $p$-values (with hypothesis tests) and standardised differences.

Cardiovascular studies had the highest reported use of c-statistics (32\% [95\% CI, 25-40\%]). For comparison, the research areas with the lowest reported use of c-statistics were urology and nephrology (9\% [95\% $\mathrm{CI}, 2-21 \%])$ and respiratory care systems (6\% [95\% CI, $1-16 \%])$. 


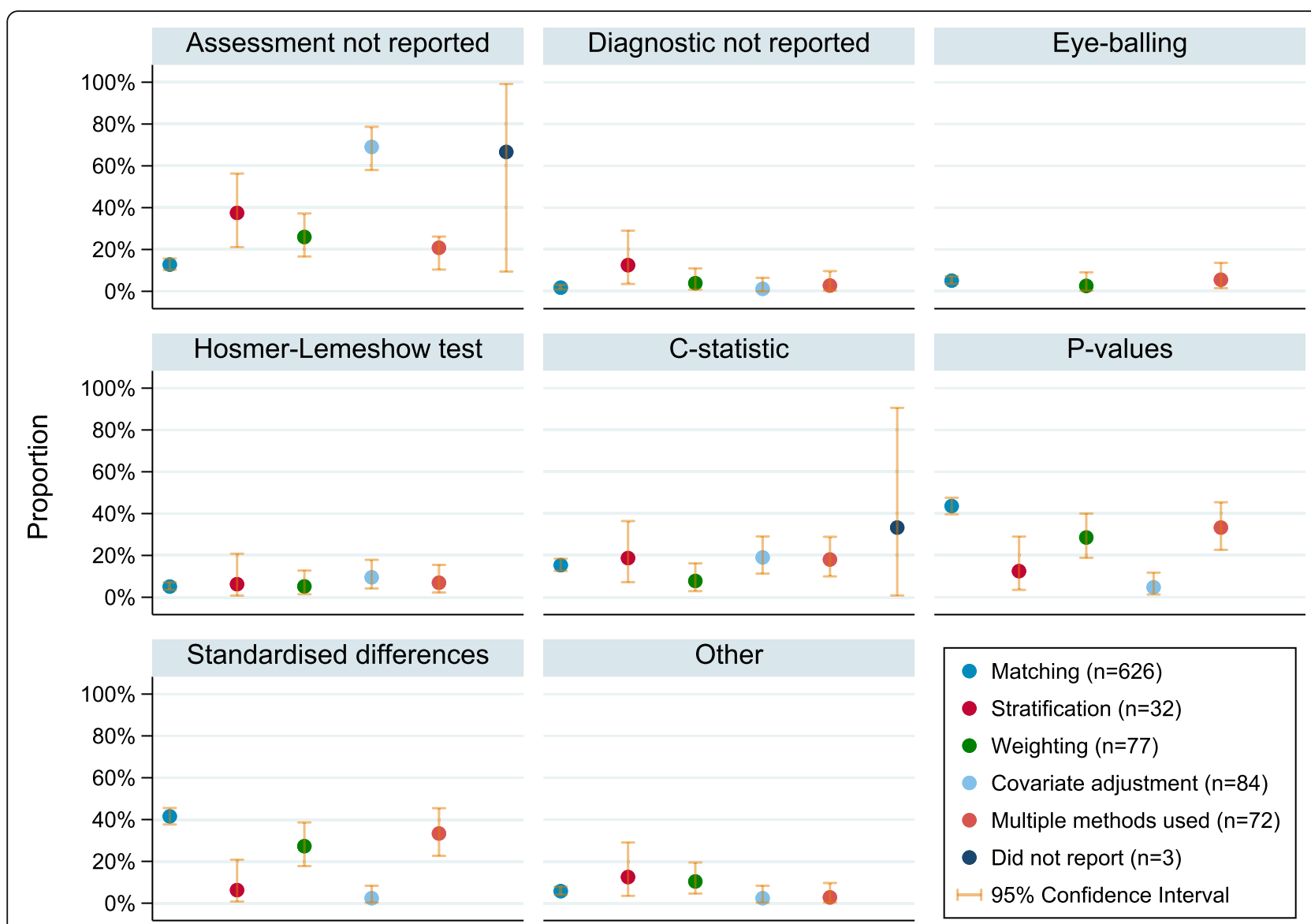

Fig. 4 The proportion (95\% Confidence Interval) of studies using each diagnostic, by propensity score method. Assessment not reported' refers to papers which did not specify whether propensity scores were assessed; 'Diagnostic not reported' refers to papers which reported that assessment took place, but not how; 'Other' includes: absolute differences, graphical approaches, post-matching c-statistic, regression, standardised bias, and variance ratios

Most research areas had approximately $25-50 \%$ of papers reporting the use of $p$-values. The area with the lowest proportion of reported use of p-values was infectious diseases (24\% [95\% CI, 13-39\%]), followed by medicine general and internal (25\% [95\% CI, 16-36\%]), cardiovascular systems (34\% [95\% CI, 26-42\%]) and peripheral vascular disease (30\% [95\% CI, 13-39\%]). Surgery studies had the highest proportion of reported use of pvalues (56\% [95\% CI, 47-65\%]).

On the other hand, surgery studies had the lowest proportion of papers reporting use of standardised differences (19\% [95\% CI, 13-28\%]). The remaining research areas had at least $26 \%$ of papers reporting use of standardised differences and the highest proportion was in studies on urology and nephrology (46\% [95\% CI, 31$61 \%])$.

\section{Comparison of diagnostics between studies using different propensity score methods}

Of the 894 studies included, 693 (78\%) used propensity score matching, 57 (6\%) used stratification, $106(12 \%)$ used covariate adjustment and $115(13 \%)$ used weighted. These percentages total to more than 100 because some studies used multiple methods. Of those that reported use of weighting, 108 used inverse-probability-of-treatment weights, 3 used standardised-mortality-ratio weighting, 1 used overlap weights and 3 did not report which weights were used.

Figure 4 compares the proportions of studies using each diagnostic by propensity score method used. Studies using covariate adjustment for the propensity score were most likely to not report whether diagnostics were used. The proportion of covariate adjustment studies failing to report this information was $69 \%$ [95\% CI, 58-79\%], whereas for stratified, weighted and matched studies, the equivalent proportions were $38 \%$ [95\% CI, 21-56\%], 26\% [95\% CI, 1737\%] and 13\% [95\% CI, 10-16\%] respectively.

While matched studies were the least likely to not report whether diagnostics were used, they were the most likely to report the use of $p$-values or standardised differences. Of the matched studies, $44 \%$ [95\% CI, 40-48\%] 
reported use of p-values, compared to 5\% [95\% CI, 1$12 \%$ ] for covariate adjustment studies, and $42 \%$ [95\% CI, $38-46 \%$ ] of matched studies reported use of standardised differences, compared to $2 \%$ [95\% CI, 0-8\%] of covariate adjustment studies.

The proportion of reported use of eye-balling, the Hosmer-Lemeshow test or the c-statistic to assess propensity scores was very similar between studies using different methods. Although there were observed differences in the use of $p$-values, standardised difference and proportion of studies not reporting assessment between studies using different methods, the F-statistic associated with the multinomial regression model of diagnostic on propensity score method had $\mathrm{p}$-value $=0.061$, indicating weak evidence for differences overall.

\section{Discussion}

A methodological review of 894 articles was conducted to assess the use of propensity score diagnostics in medical papers published in high-impact journals. Our results demonstrate that the use of propensity score diagnostics is suboptimal, even in high-impact papers, where best practice might be expected. Approximately one fifth of authors did not report the use of any diagnostic, and of those who did, hypothesis tests were the most common, despite the widespread discouragement of their use.

The problem with hypothesis tests is that they are dependent upon sample size and the hypotheses they test are not relevant. The former disadvantage is particularly problematic when matching; in the matched set an apparent improvement in balance may be due to the loss of statistical power after discarding observations. The hypotheses are irrelevant since they aim to test whether two population parameters are equal to each other, using sample data assumed to be drawn from those populations. However, when assessing propensity scores we are only interested in whether or not balance has been achieved in the sample used to estimate the treatment effects.

Additionally, our results indicated that the HosmerLemeshow test and c-statistic were also among the most commonly used diagnostics for propensity scores. These diagnostics are traditionally used for logistic regression, where the former measures goodness-of-fit. Although a significant Hosmer-Lemeshow test can identify problems with the propensity score model, it lacks power and hence a non-significant result cannot be used as evidence that a propensity score model is correct [31]. The c-statistic measures model discrimination, which, in a propensity score context, is the ability to predict treatment assignment. However, propensity scores aim to balance confounding variables, and this may not improve with improved discrimination: including variables associated with the exposure but not the outcome will improve model discrimination, but may also increase bias and variance in estimated treatment effects [32, 33]. For these reasons, we recommend against using logistic regression diagnostics for propensity scores. An exception to this recommendation, is using the c-statistic as a post-matching diagnostic [34]. If the propensity score has adequately removed imbalances between treatment groups, patient characteristics should have no association with treatment assignment in the matched set. In this case, c-statistics close to 0.5 indicate successful balance.

Standardised differences were the most commonly used diagnostic in our results, after hypothesis testing. Standardised differences are preferred because they are independent of sample size and are a property of the sample [35]. It has been suggested that a standardised difference of less than 0.1 can be considered as adequate balance [36], however this is an arbitrary threshold. The levels of acceptable imbalance will likely depend on the strength of association between covariate and outcome: stronger predictors of outcome will contribute more towards confounding bias and balance should be prioritised on these variables [37]. Additionally, it is recommended to check standardised differences in second order moments and interaction terms, since failing to do so may prevent sufficient balance being achieved on non-linear or non-additive terms [13]. If sufficient balance is not reached, authors may consider adding higher order terms, three-way interactions, transforming variables or re-categorising variables [38].

Whereas previous reviews [10,11, 18-25] did not limit their searches by journal impact factor, ours focused on high-impact journals and despite the differences in inclusion criteria there were still similarities in our results. Previous reviews [10,11,18-25] found that between 11 and $59 \%$ (20.9\% in the current study) of authors did not report the use of any diagnostic; - unfortunately, there is no evidence to suggest that this percentage is decreasing over time. Furthermore, most previous reviews [10, $11,18-20,22-25]$ also found that hypothesis tests were the most common diagnostic (the only review [21] that did not report this finding did not go into detail about which diagnostics were used). We add to the current literature by demonstrating that even in high-impact journals, where we might expect best practice to be more common, many authors are still not reporting diagnostic use, or reporting use of suboptimal diagnostics. However, comparing our results to previous reviews does suggest an increase in the use of standardised differences over time. Including this one, three reviews reported the proportion of papers using standardised differences and considered papers across all areas of medicine. The proportions and publication years (breaking down our 
results by year) in increasing order were: 4\% (19962003), 15\% (2011-2012), 34\% (2014), 35\% (2015) and $42 \%$ (2016). The increase in use of standardised differences could be a result of the recent published recommendations for their use [10-17].

Our results indicated that there were some differences in diagnostic use between different research areas. It is likely that authors will read papers within their own area and follow the apparent norm when implementing these methods in their own work. Therefore, diagnostic use could improve if tutorial papers with best practice examples were published in the leading journals of those research areas where suboptimal practice is most common. When comparing diagnostic use between studies using different propensity score methods, we observed large differences in the reported use of diagnostics in matched studies compared to the other propensity score methods: matched studies were less likely to omit reporting diagnostic use and more likely to use standardised differences. By matching subjects' results in distinct treatment groups, it is easy to compare means between groups, and so it is arguably easier to comprehend how to implement standardised differences when matching compared to the alternative methods. This could be the reason for the disparity in reported diagnostic use between matching and other methods. Fortunately there is existing guidance on how to use propensity score diagnostics when weighing [39] or using covariate adjustment [40], and well as when matching [13].

A limitation of the current study is that information regarding diagnostic use and propensity score method was only obtained manually by one author. However, we used a full-text search program to identify papers using three of the more commonly used diagnostics and thus could check for discrepancies. This revealed only a few errors; therefore, we assume that the manual search was similarly reliable for the other diagnostics. Secondly, the research areas are not necessarily categorised in the most informative way. Creating distinct and meaningful categories was challenging and there are a number of ways in which research areas could have been arbitrarily defined. We felt it was best to make use of a system already in place by using categories defined on the JCR website. Thirdly, our search may not have revealed all relevant literature. A recent study [41] found that many articles that use a particular methodology do not report its use in the title or abstract. Consequently, our review could have missed relevant articles which use propensity score methods. Moreover, by defining our search terms as "propensity score" or "propensity matched", it is possible that studies using inverse probability of treatment weighting could be underrepresented. However, the proportion of studies in our results that used this weighting method (12\%) is similar to that observed in other reviews $[11,22-25]$ on the use of propensity scores $(0-$ $14 \%)$.

Finally, regarding diagnostic use, we only collected information on which diagnostics were used and not on how they were implemented. For example, when standardised differences are used, they may be used to check balance in different samples (e.g. a matched set or a weighted sample), different thresholds for acceptable balance may be used and residual imbalance s may be handled in a variety of ways. Future research could investigate how propensity score diagnostics are implemented in the medical literature: this could help inform best practice.

\section{Conclusions}

In conclusion, the use of diagnostics in the propensity score literature remains suboptimal. Many authors are still failing to report whether diagnostics were used to assess the propensity score. However, over time we have seen some improvement. Standardised differences are currently the most widely recommended diagnostic and we have seen an increase in their use compared to previous reviews. Additionally, we identified the research areas in which suboptimal practice was most common. The propensity score literature may benefit from focused efforts to improve practice in these areas. .

\section{Supplementary information}

Supplementary information accompanies this paper at https://doi.org/10 1186/s12874-020-00994-0.

Additional file 1. Full text search string used to identify articles

Additional file 2. List of included studies

Additional file 3. Further details on Research Areas

Abbreviations

JCR: Journal Citation Report; Cl: Confidence Interval

Acknowledgements

Not applicable.

Authors' contributions

EG, TW, JS and ML contributed to the design of the study. TW conducted the search for articles, and EG and TW contributed towards data collection. EG conducted the analysis and was a major contributor in writing the manuscript. TW, JS and ML contributed towards revising the manuscript; EG, TW, JS and ML all read and approved the final version.

Funding

This work was funded by the Medical Research Council [grant number: 1789957].

\section{Availability of data and materials}

The dataset generated and analysed during the current study is available in the PropensityScoreReview repository: https://github.com/EmilyG602/ PropensityScoreReview

Ethics approval and consent to participate Not applicable. 


\section{Consent for publication}

Not applicable.

\section{Competing interests}

The authors declare that they have no competing interests.

\section{Author details}

${ }^{1}$ Centre for Epidemiology Versus Arthritis, Centre for Musculoskeletal Research, Division of Musculoskeletal and Dermatological Sciences, School of Biological Sciences, Faculty of Biology, Medicine and Health, University of Manchester, Manchester M13 9PT, UK. ${ }^{2}$ Department of Developmental Disability Neuropsychiatry, School of Psychiatry, University of New South Wales, Sydney, Australia. ${ }^{3}$ Centre for Biostatistics, Division of Population Health, Health Services Research and Primary Care, School of Health Sciences, Faculty of Biology, Medicine and Health, University of Manchester, Manchester M13 9PT, UK.

Received: 10 July 2019 Accepted: 26 April 2020

Published online: 27 May 2020

\section{References}

1. Peduzzi P, Concato J, Kemper $E$, et al. A simulation study of the number of events per variable in logistic regression analysis. J Clin Epidemiol. 1996;49: 1373-9.

2. Rosenbaum PR, Rubin DB. The central role of the propensity score in observational studies for causal effects. Biometrika. 1983;70:41-55.

3. Garrido MM, Kelley AS, Paris J, et al. Methods for constructing and assessing propensity scores. Health Serv Res. 2014;49:1701-20.

4. Rosenbaum PR, Rubin DB. Reducing bias in observational studies using subclassification on the propensity score. J Am Stat Assoc. 1984;79:516-24.

5. Austin PC. A tutorial and case study in propensity score analysis: an application to estimating the effect of in-hospital smoking cessation counseling on mortality. Multivariate Behav Res. 2011;46:119-51.

6. Austin PC, Mamdani MM. A comparison of propensity score methods: a case-study estimating the effectiveness of post-AMl statin use. Stat Med. 2006:25:2084-106.

7. D'Agostino RB Jr. Propensity score methods for bias reduction in the comparison of a treatment to a non-randomized control group. Stat Med. 1998;17:2265-81

8. Lunceford JK, Davidian M. Stratification and weighting via the propensity score in estimation of causal treatment effects: a comparative study. Stat Med. 2004;23:2937-60

9. Austin PC. An introduction to propensity score methods for reducing the effects of confounding in observational studies. Multivariate Behav Res. 2011:46:399-424.

10. Yao XI, Wang X, Speicher PJ, et al. Reporting and guidelines in propensity score analysis: a systematic review of cancer and cancer surgical studies. J Natl Cancer Inst. 2017;109:djw323.

11. Ali MS, Groenwold RHH, Belitser SV, et al. Reporting of covariate selection and balance assessment in propensity score analysis is suboptimal: a systematic review. J Clin Epidemiol. 2015;68:122-31.

12. Ali MS, Groenwold RHH, Klungel OH. Best (but oft-forgotten) practices: propensity score methods in clinical nutrition research. Am J Clin Nutr. 2016;104:247-58.

13. Austin PC. Balance diagnostics for comparing the distribution of baseline covariates between treatment groups in propensity-score matched samples. Stat Med. 2009;28:3083-107.

14. West SG, Cham H, Thoemmes F, et al. Propensity scores as a basis for equating groups: basic principles and application in clinical treatment outcome research. J Consult Clin Psychol. 2014;82:906.

15. Williamson EJ, Forbes A. Introduction to propensity scores. Respirology. 2014;19:625-35.

16. Deb S, Austin PC, Tu JV, et al. A review of propensity-score methods and their use in cardiovascular research. Can J Cardiol. 2016;32:259-65.

17. Austin PC. The use of propensity score methods with survival or time-toevent outcomes: reporting measures of effect similar to those used in randomized experiments. Stat Med. 2014;33:1242-58

18. Austin PC. A report card on propensity-score matching in the cardiology literature from 2004 to 2006: results of a systematic review. Circ Cardiovasc Qual Outcomes. 2008;1:62-7.
19. Austin PC. A critical appraisal of propensity-score matching in the medical literature between 1996 and 2003. Stat Med. 2008;27:2037-49.

20. Austin PC. Propensity-score matching in the cardiovascular surgery literature from 2004 to 2006: a systematic review and suggestions for improvement. J Thorac Cardiovasc Surg. 2007;134:1128-35.

21. Weitzen S, Lapane KL, Toledano AY, et al. Principles for modeling propensity scores in medical research: a systematic literature review. Pharmacoepidemiol Drug Saf. 2004;13:841-53.

22. Zakrison TL, Austin PC, McCredie VA. A systematic review of propensity score methods in the acute care surgery literature: avoiding the pitfalls and proposing a set of reporting guidelines. Eur J Trauma Emerg Surg. 2018;44: 385-95.

23. Thoemmes FJ, Kim ES. A systematic review of propensity score methods in the social sciences. Multivariate Behav Res. 2011;46:90-118.

24. Gayat E, Pirracchio R, Resche-Rigon M, et al. Propensity scores in intensive care and anaesthesiology literature: a systematic review. Intensive Care Med. 2010;36:1993-2003.

25. Lonjon G, Porcher R, Ergina P, et al. Potential pitfalls of reporting and bias in observational studies with propensity score analysis assessing a surgical procedure. Ann Surg. 2017;265:901-9.

26. Belitser SV, Martens EP, Pestman WR, et al. Measuring balance and model selection in propensity score methods. Pharmacoepidemiol Drug Saf. 2011; 20:1115-29.

27. Caruana E, Chevret S, Resche-Rigon M, et al. A new weighted balance measure helped to select the variables to be included in a propensity score model. J Clin Epidemiol. 2015;68:1415-22.

28. Franklin JM, Rassen JA, Ackermann D, et al. Metrics for covariate balance in cohort studies of causal effects. Stat Med. 2014;33:1685-99.

29. Stuart EA, Lee BK, Leacy FP. Prognostic score-based balance measures can be a useful diagnostic for propensity score methods in comparative effectiveness research. J Clin Epidemiol. 2013;66:S84-S90.e1.

30. Williams RL. A note on robust variance estimation for cluster-correlated data. Biometrics. 2000;56:645-6.

31. Weitzen S, Lapane K, Toledano A, et al. Weaknesses of goodness-of-fit tests for evaluating propensity score models: the case of the omitted confounder. Pharmacoepidemiol Drug Saf. 2005;14:227-38.

32. Patrick A, Schneeweiss S, Brookhart M, et al. The implications of propensity score variable selection strategies in pharmacoepidemiology: an empirical illustration. Pharmacoepidemiol Drug Saf. 2011;20:551-9.

33. Brookhart M, Schneeweiss S, Rothman KJ, et al. Variable selection for propensity score models. Am J Epidemiol. 2006;163:1149-56.

34. Westreich $D$, Cole S, Funk M, et al. The role of the c-statistic in variable selection for propensity score models. Pharmacoepidemiol Drug Saf. 2011; 20:317-20.

35. Imai K, King G, Stuart EA. Misunderstandings between experimentalists and observationalists about causal inference. J Royal Stat Soc Ser A. 2008;171: 481-502.

36. Normand S-LT, Landrum MB, Guadagnoli E, et al. Validating recommendations for coronary angiography following acute myocardial infarction in the elderly: a matched analysis using propensity scores. J Clin Epidemiol. 2001;54:387-98.

37. Ho DE, Imai K, King G, et al. Matching as nonparametric preprocessing for reducing model dependence in parametric causal inference. Polit Anal. 2007;15:199-236.

38. Lee J, Little TD. A practical guide to propensity score analysis for applied clinical research. Behav Res Ther. 2017;98:76-90.

39. Austin PC, Stuart EA. Moving towards best practice when using inverse probability of treatment weighting (IPTW) using the propensity score to estimate causal treatment effects in observational studies. Stat Med. 2015; 34:3661-79.

40. Austin PC. Goodness-of-fit diagnostics for the propensity score model when estimating treatment effects using covariate adjustment with the propensity score. Pharmacoepidemiol Drug Saf. 2008;17:1202-17.

41. Penning de Vries B, van Smeden M, Rosendaal F, et al. Title, abstract, and keyword searching resulted in poor recovery of articles in systematic reviews of epidemiologic practice. J Clin Epidemiol. 2020;121:55-61.

\section{Publisher's Note}

Springer Nature remains neutral with regard to jurisdictional claims in published maps and institutional affiliations. 